\title{
Lower bounds for resonance counting functions for Schrödinger operators with fixed sign potentials in even dimensions
}

\author{
T. J. Christiansen
}

\begin{abstract}
If $d$ is even, the resonances of the Schrödinger operator $-\Delta+V$ on $\mathbb{R}^{d}$ with $V \in L_{\text {comp }}^{\infty}\left(\mathbb{R}^{d}\right)$ are points on $\Lambda$, the logarithmic cover of $\mathbb{C} \backslash\{0\}$. We show that for fixed sign potentials $V$ and for $m \in \mathbb{Z} \backslash\{0\}$, the resonance counting function for the $m$ th sheet of $\Lambda$ has maximal order of growth.
\end{abstract}

Mathematics Subject Classification (2010). Primary: 35P25; Secondary: 81U05, 47A40.

Keywords. Resonance, Schrödinger operator, scattering theory.

\section{Introduction}

This paper proves some optimal lower bounds on the order of growth of resonancecounting functions for certain Schrödinger operators in even-dimensional Euclidean space. The resonances associated to the Schrödinger operator $-\Delta+V$, with potential $V \in L_{\text {comp }}^{\infty}\left(\mathbb{R}^{d}\right)$, lie on $\Lambda$, the logarithmic cover of $\mathbb{C} \backslash\{0\}$, if $d$ is even. The main result of this paper is that for scattering by a fixed sign, compactly supported potential $V$ the resonance counting function for the $m$ th sheet of $\Lambda$ has maximal order of growth for any $m \in \mathbb{Z} \backslash\{0\}$. Though the results of [7] show that there are many potentials with resonance counting functions for the $m$ th sheet having maximal order of growth, the techniques of [7] do not give a way of identifying those potentials other than those which are scalar multiples of the characteristic function of a ball. For comparison, in odd dimensions $d \geq 3$ the only specific realvalued potentials $V \in L_{\text {comp }}^{\infty}\left(\mathbb{R}^{d}\right)$ which are known to have resonance-counting function with optimal order of growth are certain radial potentials [31], though in that case asymptotics are known (see [31] and [26, 10]). 
Let $V \in L_{\text {comp }}^{\infty}\left(\mathbb{R}^{d}\right)$ and let $\Delta \leq 0$ denote the Laplacian on $\mathbb{R}^{d}$. We denote the resolvent $R_{V}(\lambda)=\left(-\Delta+V-\lambda^{2}\right)^{-1}$ for $\lambda$ in the "physical space," $0<\arg \lambda<\pi$. With at most a finite number of exceptional values of $\lambda, R_{V}(\lambda)$ is a bounded operator on $L^{2}\left(\mathbb{R}^{d}\right)$ for $\lambda$ in this region. It is well known that for $\chi \in L_{\text {comp }}^{\infty}\left(\mathbb{R}^{d}\right), \chi R_{V}(\lambda) \chi$ has a meromorphic continuation to $\mathbb{C}$ when $d$ is odd and to $\Lambda$, the logarithmic cover of $\mathbb{C} \backslash\{0\}$, when $d$ is even (e.g. [19, Chapter 2]). In either case, the resonances are defined to be the poles of $\chi R_{V}(\lambda) \chi$ when $\chi$ is chosen to satisfy $\chi V \equiv V$. The fact that when $d$ is even the resonances lie on $\Lambda$ makes them generally more difficult to study in the even-dimensional case than in the odd-dimensional case.

A point on $\Lambda$ can be described by its modulus and argument, where we do not identify points which have arguments differing by nonzero integral multiples of $2 \pi$. Thus the physical half plane corresponds to

$$
\Lambda_{0} \stackrel{\text { def }}{=}\{\lambda \in \Lambda: 0<\arg \lambda<\pi\} .
$$

Likewise, for $m \in \mathbb{Z}$ we may define the $m$ th sheet to be

$$
\Lambda_{m} \stackrel{\text { def }}{=}\{\lambda \in \Lambda: m \pi<\arg \lambda<(m+1) \pi\}
$$

which is homeomorphic with the physical region and can be identified with the upper half plane when convenient.

Vodev [28, 29], following earlier work of Intissar [13] studied the resonance counting function $n_{V}(r, a)$, defined to be the number of resonances (counted with multiplicity, here and everywhere) with norm at most $r$ and argument between $-a$ and $a$. He showed that there is a constant $C$ which depends on $V$ but not on $r$ or $a$ so that

$$
\left.n_{V}(r, a) \leq C a\left(r^{d}+(\log a)^{d}\right)\right), \quad \text { for } r, a>1 .
$$

The most general lower bound known is due to Sá Barreto ([22], $d \geq 4)$ and Chen ([3], d=2):

$$
\lim \sup _{r \rightarrow \infty} \frac{\#\left\{\lambda_{j}: \text { pole of } R_{V}(\lambda) \text { with } \frac{1}{r} \leq\left|\lambda_{j}\right| \leq r,\left|\arg \lambda_{j}\right| \leq \log r\right\}}{(\log r)(\log \log r)^{-p}}=\infty
$$

for all $p>1$, for any nontrivial $V \in C_{c}^{\infty}\left(\mathbb{R}^{d} ; \mathrm{R}\right)$. This follows the earlier work of [23]. We note that the assumption that the potential is real-valued is crucial here. There are explicit examples of nontrivial complex-valued potentials $V \in L_{\text {comp }}^{\infty}\left(\mathbb{R}^{d}\right)$ which can be chosen to be smooth so that the corresponding Schrödinger operator $-\Delta+V$ has neither eigenvalues nor resonances $[2,5,6]$. 
For $m \in \mathbb{Z}$, let $n_{m}(r)=n_{m, V}(r)$ be the number of resonances of $-\Delta+V$ which both lie on $\Lambda_{m}$ and have norm at most $r$. We call this the resonance counting function for the $m$ th sheet. It follows from Vodev's result that $n_{m}(r)=O\left(r^{d}\right)$ as $r \rightarrow \infty$. On the other hand, lower bounds have proved more elusive, as demonstrated by the difference between the upper bound and the lower bound (1.1). The results of [7, Theorem 1.1] show that "generically" for potentials $V \in L_{\text {comp }}^{\infty}\left(\mathbb{R}^{d}\right)$, $m \in \mathbb{Z} \backslash\{0\}$,

$$
\lim \sup _{r \rightarrow \infty} \frac{\log n_{m, V}(r)}{\log r}=d .
$$

However, the result of [7] is nonconstructive in the sense that other than potentials which are nonzero positive scalar multiples of the characteristic function of a ball and those complex-valued potentials which are isoresonant with them [6], that paper does not give a way of identifying the particular potentials for which (1.2) holds.

The main result of this paper is the following theorem.

Theorem 1.1. Let $d$ be even. Suppose $V \in L_{\text {comp }}^{\infty}\left(\mathbb{R}^{d}\right)$ with $V$ bounded below by $\epsilon \chi_{B}$, where $\epsilon>0$ and $\chi_{B}$ is the characteristic function of a nontrivial ball $B$. Then for any nonzero $m \in \mathbb{Z}$,

$$
\lim \sup _{r \rightarrow \infty} \frac{\log n_{m, \pm V}(r)}{\log r}=d .
$$

We note that by Vodev's result $d$ is the maximum value this limit can attain. The limit

$$
\lim \sup _{r \rightarrow \infty} \frac{\log n_{m, \pm V}(r)}{\log r}
$$

is called the order (or order of growth) of $n_{m, \pm V}(r)$. When this limit is $d$, we say that the $m$ th counting function has maximal order of growth.

Theorem 1.1, when combined with [7, Theorem 3.8], has the following theorem as an immediate corollary.

Theorem 1.2. Let $d$ be even, and $K \subset \mathbb{R}^{d}$ be a compact set with nonempty interior. Let $F$ denote either $\mathbb{R}$ or $\mathbb{C}$. Then for $m \in \mathbb{Z}, m \neq 0$, the set

$$
\left\{V \in C^{\infty}(K ; F): \lim \sup _{r \rightarrow \infty} \frac{\log n_{m, V}(r)}{\log r}=d\right\}
$$

is dense in $C^{\infty}(K ; F)$.

The paper [7] proves a similar theorem, but for $L^{\infty}$ potentials rather than $C^{\infty}$ potentials. For the case of $o d d$ dimension $d \geq 3$, the analog of Theorem 1.2 was proved in [4]. A stronger result holds in dimension $d=1$, see [30] or [11, 21, 24]. 
Theorem 1.1 may be compared with other results for fixed-sign potentials. In [8] it is shown that in even dimensions there are no "pure imaginary" resonances for positive potentials, and on each sheet $\Lambda_{m}$ of $\Lambda$, only finitely many for negative potentials. In contrast, in the odd-dimensional case Lax-Phillips [16] and Vasy [27] proved that for potentials of fixed sign the number of pure imaginary resonances of norm at most $r$ grows at least like $c r^{d-1}$ for some $c>0$ when $r$ is sufficiently large. Both [16] and [27] use a monotonicity property for potentials of fixed sign. This paper also uses a monotonicity property, though it is more closely related to one used in [4]. Also important here are some results from one-dimensional complex analysis, more delicate than the corresponding complex-analytic arguments from [4].

We end this introduction with a brief sketch of the proof of Theorem 1.1. We shall reduce the problem of studying the large $r$ behavior of $n_{m, \pm V}(r)$ to that of studying the zeros of a function holomorphic in $\overline{\Lambda_{0}}$.

In Section 2 we prove Proposition 2.4, a preliminary, complex-analytic, result. This provides an upper bound on the norm of a function $f$ holomorphic in the set

$$
\Omega=\{z \in \mathbb{C}:|z| \geq 1, \operatorname{Im} z \geq 0\}
$$

when we have some control on both the behavior of $f(z)$ for large $|z|, z \in \mathbb{R}$ and on the order of growth of its zero counting function in $\Omega$.

Let $m \in \mathbb{Z} \backslash\{0\}$. To study the resonances of $-\Delta \pm V$ on $\Lambda_{m}$ we introduce a scalar function $F_{m, \pm V}$ defined on $\overline{\Lambda_{0}}$. The zeros of $F_{m, \pm V}$ on $\Lambda_{0}$ correspond (with at most finitely many exceptions) to the resonances of $-\Delta \pm V$ on $\Lambda_{m}$. The function $F_{m, \pm V}$ is meromorphic on $\Lambda_{0}$, with at most finitely many poles there. The definition of $F_{m, \pm V}$ in Section 3 uses familiar ideas from the study of resonances.

The main result of Section 4 is a lower bound on $\left|F_{m, \pm V}\left(\sigma e^{i \pi / 2}\right)\right|$ when $\sigma \rightarrow \infty$ and $V \geq \epsilon \chi_{B}$. It is here that we use a monotonicity argument. The proof proceeds by contradiction. In Section 5 we show that when $V \geq \epsilon \chi_{B}$, the assumption

$$
\lim \sup _{r \rightarrow \infty} \frac{\log n_{m, \pm V}(r)}{\log r}<d
$$

together with the lower bound on $\left|F_{m, \pm V}\left(\sigma e^{i \pi / 2}\right)\right|$ as $\sigma \rightarrow \infty$ from Section 4 produces a contradiction to Proposition 2.4.

Acknowledgments. The author gratefully acknowledges the partial support of the NSF under grant DMS 1001156, and thanks the referees for helpful comments. 


\section{Some Complex Analysis}

The main result of this section is Proposition 2.4, which, roughly speaking, controls the growth of a function $f$ analytic in a half plane in terms of the growth of the counting function for the zeros of $f$ in the half plane and the behavior of $f$ on the boundary of the half plane.

Both the statement and the proof of the following lemma bear some resemblance to those for Carathéodory's inequality for the disk. The estimate we obtain here is likely a crude one, but suffices for our purposes.

Lemma 2.1. Let $f$ be analytic in a neighborhood of

$$
\Omega_{R} \stackrel{\text { def }}{=}\{z \in \mathbb{C}: 1 \leq|z| \leq R, \operatorname{Im} z \geq 0\},
$$

$\rho>0$, and for $x \in \mathbb{R} \cap \Omega_{R},|f(x)| \leq C_{0}|x|^{\rho}$ for some constant $C_{0}>0$. Set

$$
M=\max _{|z|=1, z \in \Omega_{R}}|f(z)|
$$

and define

$$
A(R)=\max \left(C_{0} R^{\rho}, M R^{\rho}, \max _{z \in \Omega_{R}} \operatorname{Re} f(z)\right) .
$$

Then if $1<r<R$ and $z \in \Omega_{R}$ with $|z|=r$, then $|f(z)| \leq \frac{2 r^{\rho}}{R^{\rho}-r^{\rho}} A(R)$.

Proof. Set

$$
g(z)=\frac{1}{z^{\rho}} \frac{f(z)}{2 A(R)-f(z)}
$$

which is analytic in a neighborhood of $\Omega_{R}$. We bound $|g|$ on the boundary of $\Omega_{R}$. If $z \in \Omega_{R}$ has $|z|=R$, then

$$
|g(z)| \leq \frac{1}{R^{\rho}} \frac{|f(z)|}{|2 A(R)-f(z)|} \leq \frac{|f(z)|}{R^{\rho}|f(z)|}=\frac{1}{R^{\rho}} .
$$

Notice that if $x \in \Omega_{R} \cap \mathbb{R}$,

$$
|g(x)| \leq \frac{1}{|x|^{\rho}} \frac{C_{0}|x|^{\rho}}{R^{\rho} C_{0}} \leq \frac{1}{R^{\rho}} .
$$

Moreover, if $z \in \Omega_{R}$ has $|z|=1$, since $A(R) \geq|f(z)| R^{\rho},|g(z)| \leq 1 / R^{\rho}$. Thus, by the maximum principle $|g(z)| \leq 1 / R^{\rho}$ for all $z \in \Omega_{R}$. 
Suppose $z \in \Omega_{R}$ with $|z|=r, 1<r<R$. Then

$$
|f(z)| \leq|z|^{\rho}|2 A(R)-f(z)| R^{-\rho} \leq r^{\rho}(2 A(R)+|f(z)|) R^{-\rho} .
$$

Rearranging, we find

$$
\left(R^{\rho}-r^{\rho}\right)|f(z)| \leq 2 r^{\rho} A(R),
$$

or

$$
|f(z)| \leq \frac{2 r^{\rho}}{R^{\rho}-r^{\rho}} A(R) .
$$

Lemma 2.2. Let

$$
\Omega=\{z: \operatorname{Im} z \geq 0,|z| \geq 1\}
$$

and suppose $f$ is analytic in a neighborhood of $\Omega$, and there are constants $\rho_{0}$, $C_{0}$, so that

$$
|f(z)| \leq C_{0} \exp \left(C_{0}|z|^{\rho_{0}}\right) \quad \text { for all } z \in \Omega .
$$

Suppose there are constants $C_{1}, \rho>0$ so that

$$
\left|\int_{1}^{x} f^{\prime}(t) / f(t) d t\right| \leq C_{1}|x|^{\rho}, \text { for all } x>1,
$$

and

$$
\left|\int_{x}^{-1} f^{\prime}(t) / f(t) d t\right| \leq C_{1}|x|^{\rho}, \quad \text { for all } x<-1 .
$$

If, in addition, $f$ does not vanish in $\Omega$, then there is a constant $C_{3}$ so that

$$
|f(z)| \leq C_{3} \exp \left(C_{3}|z|^{\rho}\right) \quad \text { for all } z \in \Omega .
$$

Proof. In the proof we shall denote by $C$ a constant the value of which may change from line to line without comment.

Since $f$ is nonvanishing in $\Omega$, there is a function $g$ analytic on $\Omega$ so that $\exp g(z)=f(z)$. Since $g^{\prime}(z)=f^{\prime}(z) / f(z)$,

$$
g(x)-g(1)=\int_{1}^{x} \frac{f^{\prime}(t)}{f(t)} d t \quad \text { if } x>1
$$

so that $|g(x)| \leq C|x|^{\rho}+|g(1)|$ when $x \geq 1$ for some constant $C$. A similar argument gives a similar bound for $x \leq-1$. 
We now assume $\rho<\rho_{0}$ since otherwise there is nothing to prove. We give a bound on the growth of $g$ at infinity which is more than adequate to allow us to apply a version of the Phragmén-Lindellöf theorem as we will below. Since $\operatorname{Re} g(z)=\log |f(z)|$, for all $z \in \Omega, \operatorname{Re} g(z) \leq C\left(1+|z|^{\rho_{0}}\right)$. Applying Lemma 2.1, we find that $|g(z)| \leq C\left(1+|z|^{\rho_{0}}\right)$ for all $z \in \Omega$.

Consider the function $h(z)=g(z) /(i+z)^{\rho}$. This is an analytic function in a neighborhood of $\Omega$ and is bounded on $\partial \Omega$. Thus, by a version of the PhragménLindellöf theorem (proved, for example, by an easy modification of the proof of [9, Corollary VI.4.2] using [9, Theorem VI.4.1]), $h$ is bounded in $\Omega$. This implies that for $z \in \Omega,|f(z)|=\exp (\operatorname{Re} g(z)) \leq \exp \left(C(1+|z|)^{\rho}\right)$ for some constant $C$.

We shall use the notation

$$
E_{0}(z)=1-z
$$

and

$$
E_{p}(z)=(1-z) \exp \left(z+z^{2} / 2+\cdots+z^{p} / p\right) \quad \text { for } p \in \mathbb{N}
$$

for a canonical factor. The proof of the following lemma bears many similarities to proofs for estimates of canonical products; see for example [17, Lemma I.4.3].

Lemma 2.3. Let $\left\{a_{j}\right\} \subset \mathbb{C}$ be a set of not necessarily distinct points in the open upper half plane, with $\left|a_{1}\right| \leq\left|a_{2}\right| \leq \ldots$ and suppose for some constants $C_{0}$ and $\rho$

$$
n(r) \stackrel{\text { def }}{=} \#\left\{j:\left|a_{j}\right| \leq r\right\} \leq C_{0} r^{\rho} \quad \text { when } r \geq 1 .
$$

Suppose $\rho>0$ is not an integer, let $p$ be the greatest integer less than $\rho$, and set

$$
f(z)=\prod_{n=1}^{\infty} \frac{E_{p}\left(z / \overline{a_{n}}\right)}{E_{p}\left(z / a_{n}\right)} .
$$

Then for $x \in \mathbb{R}$

$$
\left|\int_{0}^{x} \frac{f^{\prime}(t)}{f(t)} d t\right|=O\left(|x|^{\rho}\right)
$$

as $|x| \rightarrow \infty$.

Proof. We note first that our assumption on $n(r)$ ensures that the canonical products converge, so that $f$ is a meromorphic function on $\mathbb{C}$. Moreover, by assumption $f$ has neither poles nor zeros on the real line. 
A computation shows that $E_{p}^{\prime}(z) / E_{p}(z)=-z^{p} /(1-z)$. Thus

$$
\frac{f^{\prime}(x)}{f(x)}=\sum_{n=1}^{\infty}\left(\frac{\left(x / \overline{a_{n}}\right)^{p}}{x-\overline{a_{n}}}-\frac{\left(x / a_{n}\right)^{p}}{x-a_{n}}\right) .
$$

Let $a \in \mathbb{C}, \operatorname{Im} a>0, t \in \mathbb{R}$. Then

$$
\begin{aligned}
\frac{(t / \bar{a})^{p}}{t-\bar{a}}-\frac{(t / a)^{p}}{t-a} & =\frac{1}{|a|^{2 p}} t^{p}\left(\frac{a^{p}(t-a)-\bar{a}^{p}(t-\bar{a})}{|t-a|^{2}}\right) \\
& =2 i \frac{1}{|a|^{2 p}} t^{p}\left(\frac{t \operatorname{Im}\left(a^{p}\right)-\operatorname{Im}\left(a^{p+1}\right)}{|t-a|^{2}}\right) .
\end{aligned}
$$

Now set $a=\alpha+i \beta, \beta>0$, and note that $\left|\operatorname{Im} a^{p}\right| \leq p \beta|a|^{p-1}$. Thus for $x \in \mathbb{R}$

$$
\begin{aligned}
& \left|\int_{0}^{x}\left(\frac{(t / \bar{a})^{p}}{t-\bar{a}}-\frac{(t / a)^{p}}{t-a}\right) d t\right| \\
& \quad \leq \frac{2}{|a|^{p+1}}\left|\int_{0}^{x} \frac{p|t|^{p+1} \beta+(p+1)|a||t|^{p} \beta}{(t-\alpha)^{2}+\beta^{2}} d t\right| .
\end{aligned}
$$

Now for $q>0$

$$
\begin{aligned}
\int_{0}^{x} & \frac{t^{q} \beta}{(t-\alpha)^{2}+\beta^{2}} d t \\
& =x^{q} \arctan \left(\frac{(x-\alpha)}{\beta}\right)-q \int_{0}^{x} t^{q-1} \arctan \left(\frac{(t-\alpha)}{\beta}\right) d t .
\end{aligned}
$$

Using that for $s \in \mathbb{R},|\arctan s|<\pi / 2$, we find that for $|x|>1$

$$
\left|\int_{0}^{x} \frac{t^{q} \beta}{(t-\alpha)^{2}+\beta^{2}} d t\right| \leq C|x|^{q}
$$

for some constant $C$, independent of $\alpha$ and $\beta$.

To prove the lemma, we will split $\left\{a_{j}\right\}$ into two sets, depending on the relative size of $\left|a_{j}\right|$ and $2|x|$. For $\left|a_{j}\right| \leq 2|x|$, we first note that

$$
p \int_{0}^{x} \frac{\beta t^{p+1}}{(t-\alpha)^{2}+\beta^{2}} d t=p \beta \int_{0}^{x} t^{p-1}\left(1+\frac{2 \alpha t-\left(\alpha^{2}+\beta^{2}\right)}{(t-\alpha)^{2}+\beta^{2}}\right) d t
$$

and use (2.3) and (2.4) to get

$$
\begin{aligned}
& \sum_{\left|a_{j}\right| \leq 2|x|}\left|\int_{0}^{x}\left(\frac{\left(t / \overline{a_{j}}\right)^{p}}{t-\overline{a_{j}}}-\frac{\left(t / a_{j}\right)^{p}}{t-a_{j}}\right) d t\right| \\
& \quad \leq C \sum_{\left|a_{j}\right| \leq 2|x|}\left(|x|^{p}\left|a_{j}\right|^{-p}+|x|^{p-1}\left|a_{j}\right|^{-p+1}\right) .
\end{aligned}
$$


Since

$$
\begin{aligned}
\sum_{1 \leq\left|a_{j}\right| \leq r}\left|a_{j}\right|^{q} & =\int_{1}^{r} t^{q} d n(t) \\
& =r^{q} n(r)-n(1)-\int_{1}^{r} q t^{q-1} n(t) d t
\end{aligned}
$$

applying our upper bound on $n(r)$ we get from (2.5)

$$
\sum_{\left|a_{j}\right| \leq 2|x|}\left|\int_{0}^{x}\left(\frac{\left(t / \overline{a_{j}}\right)^{p}}{t-\overline{a_{j}}}-\frac{\left(t / a_{j}\right)^{p}}{t-a_{j}}\right) d t\right| \leq C\left(|x|^{\rho}+1\right) .
$$

Now we bound the contribution of the $a_{j}$ with $\left|a_{j}\right|>2|x|$. For this we use (2.2) more directly. Here

$$
\begin{aligned}
& \sum_{\left|a_{j}\right|>2|x|}\left|\int_{0}^{x}\left(\frac{\left(t / \overline{a_{j}}\right)^{p}}{t-\overline{a_{j}}}-\frac{\left(t / a_{j}\right)^{p}}{t-a_{j}}\right) d t\right| \\
& \quad=2 \sum_{\left|a_{j}\right|>2|x|}\left|\int_{0}^{x} \frac{1}{\left|a_{j}\right|^{2 p}} t^{p}\left(\frac{t \operatorname{Im}\left(a_{j}^{p}\right)-\operatorname{Im}\left(a_{j}^{p+1}\right)}{\left|t-a_{j}\right|^{2}}\right) d t\right| \\
& \quad \leq C \sum_{\left|a_{j}\right|>2|x|}\left|\int_{0}^{x} \frac{1}{\left|a_{j}\right|^{2 p}}\left(\frac{|t|^{p+1}\left|a_{j}\right|^{p}+|t|^{p}\left|a_{j}\right|^{p+1}}{\left|a_{j}\right|^{2}}\right) d t\right| \\
& \quad \leq C \sum_{\left|a_{j}\right|>2|x|}\left(|x|^{p+2}\left|a_{j}\right|^{-p-2}+|x|^{p+1}\left|a_{j}\right|^{-p-1}\right) .
\end{aligned}
$$

Applying the analog of (2.6) and using the upper bound on $n(r)$ we obtain

$$
\sum_{\left|a_{j}\right|>2|x|}\left|a_{j}\right|^{-q} \leq C|x|^{\rho-q}
$$

provided $q>\rho$, giving us

$$
\sum_{\left|a_{j}\right|>2|x|}\left|\int_{0}^{x}\left(\frac{\left(t / \overline{a_{j}}\right)^{p}}{t-\overline{a_{j}}}-\frac{\left(t / a_{j}\right)^{p}}{t-a_{j}}\right) d t\right| \leq C|x|^{\rho} .
$$

Combined with (2.7), this completes the proof of the lemma. 
Proposition 2.4. Let $f$ be a function analytic in a neighborhood of

$$
\Omega=\{z: \operatorname{Im} z \geq 0,|z| \geq 1\} .
$$

Suppose $f$ does not vanish on $\mathbb{R} \cap \Omega$, and let $n(r)$ be the number of zeros of $f$ in $\{z: \operatorname{Im} z \geq 0,1 \leq|z| \leq r\}$ counted with multiplicity. Suppose that that there are constants $C_{0}$ and $\rho>0$, $\rho$ not an integer, so that

$$
n(r) \leq C_{0}\left(1+r^{\rho}\right)
$$

and

$$
\left|\frac{f^{\prime}(x)}{f(x)}\right| \leq C_{0}\left(1+|x|^{\rho-1}\right) \quad \text { for all } x \in \mathbb{R} \text { with }|x| \geq 1 .
$$

Suppose in addition that there are some constants $\rho_{1}, C_{1}$ so that $\log |f(z)| \leq$ $C_{1}\left(1+|z|^{\rho_{1}}\right)$ for all $z \in \Omega$. Then there is a constant $C$ so that $|f(z)| \leq C e^{|z|^{\rho}}$ for $z \in \Omega$.

Proof. We will assume $\rho_{1}>\rho$ as otherwise there is nothing to prove.

We prove this proposition by constructing a function to which we can apply Lemma 2.2. Let $p$ denote the greatest integer less than $\rho$, and $\left\{a_{j}\right\}$ the zeros of $f$ in $\Omega$, repeated according to multiplicity, with $\left|a_{1}\right| \leq\left|a_{2}\right| \leq \ldots$. Set

$$
h(z)=\frac{f(z) g_{1}(z)}{g_{2}(z)},
$$

where

$$
g_{1}(z)=\prod_{n=1}^{\infty} E_{p}\left(z / \overline{a_{n}}\right) \text { and } g_{2}(z)=\prod_{n=1}^{\infty} E_{p}\left(z / a_{n}\right) .
$$

Note that $h$ is analytic in $\Omega$ and does not vanish there.

As an intermediate step we show that $\log |h(z)| \leq C|z|^{\rho_{1}}$ for all $z \in \Omega$. Recall we have assumed $\rho_{1}>\rho$. Here and below $C$ is a finite constant which may change from line to line. If $x \in \mathbb{R}, 1 \leq|x|$, then $\log |h(x)|=\log |f(x)| \leq C_{0}\left(1+|x|^{\rho_{1}}\right)$. Moreover, from estimates on canonical products,

$$
\log \left|g_{j}(z)\right| \leq C\left(1+|z|^{\rho}\right), \quad j=1,2
$$

for some constant $C$, see [17, Lemma I.4.3].

As is shown in the proof of [17, Theorem I.12], given $R>0$ and $0<\delta<1$ there are $r_{j} \in\left[R, R(1-\delta)^{-1}\right]$, (depending on $R$ and $\delta$ ) so that for all $z \in \mathbb{C}$ with $|z|=r_{j}$,

$$
\log \left|g_{j}(z)\right| \geq-\left(2+\log \frac{12 e}{\delta}\right) \log \max _{|z|=2 e R(1-\delta)^{-1}}\left|g_{j}(z)\right|, \quad \text { for } j=1,2 .
$$


Using (2.8), this gives

$$
\log \left|g_{j}(z)\right| \geq-C_{\delta, j}\left(1+\left(R(1-\delta)^{-1}\right)^{\rho}\right), \quad|z|=r_{j}, j=1,2 .
$$

To aid in notation, we set $\Omega_{R}=\{z \in \mathbb{C}: \operatorname{Im} z \geq 0$ and $1 \leq|z| \leq R\}$.

Now fix $\delta>0, \delta<1$. Given any $R>1$, we can find an $r_{2} \in\left[R, R(1-\delta)^{-1}\right]$ as above so that (2.10) holds for $j=2$. Then using that

$$
\max _{z \in \Omega_{R}} \log |h(z)| \leq \max _{z \in \Omega_{r_{2}}} \log |h(z)|=\max _{z \in \partial \Omega_{r_{2}}} \log |h(z)|,
$$

our assumptions on $f$, and (2.10) we find for any $R>1$

$$
\max _{z \in \Omega_{R}} \log |h(z)| \leq C_{\delta}\left(1+\left(R(1-\delta)^{-1}\right)^{\rho_{1}}\right)+C\left(1+R^{\rho}\right) \leq \tilde{C}_{\delta}\left(1+R^{\rho_{1}}\right) .
$$

For $x \in \mathbb{R},|x| \geq 1$,

$$
\frac{h^{\prime}(x)}{h(x)}=\frac{f^{\prime}(x)}{f(x)}+\frac{\left(g_{1} / g_{2}\right)^{\prime}(x)}{g_{1}(x) / g_{2}(x)} .
$$

By applying our assumptions on $f$ and Lemma 2.3, we find that for $x>1$,

$$
\left|\int_{1}^{x} h^{\prime}(t) / h(t) d t\right|=O\left(x^{\rho}\right),
$$

and likewise for $x<-1$,

$$
\left|\int_{x}^{-1} h^{\prime}(t) / h(t) d t\right|=O\left(|x|^{\rho}\right) .
$$

By Lemma 2.2, there is a constant $C$ so that

$$
\log |h(z)| \leq C\left(1+|z|^{\rho}\right), \text { when } z \in \Omega .
$$

Now we write $f(z)=g_{2}(z) h(z) / g_{1}(z)$, holomorphic in a neighborhood of $\Omega$. Given $R>1$ and $\delta$ satisfying $0<\delta<1$, as above we choose $r_{1} \in\left[R, R(1-\delta)^{-1}\right]$ so that (2.10) holds for $g_{1}$. Using in addition (2.8) and (2.11), we find there is a constant so that

$$
\log |f(z)| \leq C\left(1+\left(R(1-\delta)^{-1}\right)^{\rho}\right) \text { for }|z|=r_{1}, \operatorname{Im} z \geq 0 .
$$

As in the proof of the bound on $h$, since $|h(x)|=|f(x)|$ for $x \in \mathbb{R} \cap \Omega_{R}$, we find then from the maximum principle that there is a constant $C$ so that

$$
\max _{z \in \Omega_{R}} \log |f(z)| \leq C\left(1+R^{\rho}\right) .
$$




\section{A scalar function having zeros at the poles of the resolvent}

We recall the derivation of some identities commonly used in the study of resonances for Schrödinger operators. Let $V \in L_{\text {comp }}^{\infty}\left(\mathbb{R}^{d}\right)$ and let $d \geq 2$ be even. There is no need to make an assumption on the sign of $V$ here. We recall the notation $R_{V}(\lambda)=\left(-\Delta+V-\lambda^{2}\right)^{-1}$ when $\lambda \in \Lambda_{0}$. For such $\lambda,\left(-\Delta+V-\lambda^{2}\right) R_{0}(\lambda)=$ $I+V R_{0}(\lambda)$ and by meromorphic continuation,

$$
R_{0}(\lambda)=R_{V}(\lambda)\left(I+V R_{0}(\lambda)\right), \quad \lambda \in \Lambda .
$$

Thus $R_{V}(\lambda)$ has a pole if and only if $I+V R_{0}(\lambda)$ has a zero, and the multiplicities agree. Writing $V^{1 / 2}=V /|V|^{1 / 2}$ with the convention that $V^{1 / 2}=0$ outside the support of $V$, we see that $I+V R_{0}(\lambda)$ has a zero if and only if $I+V^{1 / 2} R_{0}(\lambda)|V|^{1 / 2}$ has a zero. Consequently, $I+V^{1 / 2} R_{0}(\lambda)|V|^{1 / 2}$ is invertible for all but a finite number of points in $\overline{\Lambda_{0}}$. Thus, if $m \in \mathbb{Z}, \lambda \in \overline{\Lambda_{0}}$,

$$
\begin{aligned}
I+ & V^{1 / 2} R_{0}\left(e^{i m \pi} \lambda\right)|V|^{1 / 2} \\
= & \left(I+V^{1 / 2} R_{0}(\lambda)|V|^{1 / 2}\right) \\
& \quad\left(I+\left(I+V^{1 / 2} R_{0}(\lambda)|V|^{1 / 2}\right)^{-1} V^{1 / 2}\left(R_{0}\left(e^{i m \pi} \lambda\right)-R_{0}(\lambda)\right)|V|^{1 / 2}\right) .
\end{aligned}
$$

But when $d$ is even

$$
R_{0}\left(e^{i m \pi} \lambda\right)-R_{0}(\lambda)=i m T(\lambda)
$$

with

$$
(T(\lambda) f)(x)=\alpha_{d} \lambda^{d-2} \int_{\mathbb{R}^{d}} \int_{\mathbb{S}^{d-1}} e^{i \lambda(x-y) \cdot \omega} f(y) d \omega d y
$$

for $f \in L_{\text {comp }}^{2}\left(\mathbb{R}^{d}\right)$, with $\alpha_{d}=(2 \pi)^{1-d} / 2$; see [19, (1.32)]. Moreover, $V^{1 / 2} T(\lambda)|V|^{1 / 2}$ is trace class. Thus, with at most a finite number of exceptions, the poles of $R_{V}\left(e^{i m \pi} \lambda\right)$ with $\lambda \in \Lambda_{0}$ correspond, with multiplicity, to the zeros of

$$
F_{m, V}(\lambda) \stackrel{\operatorname{def}}{=} \operatorname{det}\left(I+i m\left(I+V^{1 / 2} R_{0}(\lambda)|V|^{1 / 2}\right)^{-1} V^{1 / 2} T(\lambda)|V|^{1 / 2}\right)
$$

in $\Lambda_{0}$. Similar functions were used, for example, in $[11,12,8]$. 


\section{Lower bounds on $F_{m, \pm V}(i \sigma)$ when $V$ has fixed sign}

In the remainder of this paper we assume $d \geq 2$ is even.

Let $V \geq 0, V \in L_{\text {comp }}^{\infty}\left(\mathbb{R}^{d}\right)$. In this section we study the function $F_{m, \pm V}$ from (3.2). For $\sigma \in \mathbb{R}_{+}$, we shall use the shorthand $i \sigma$ to denote the point in the physical region with norm $\sigma$ and argument $\pi / 2$. Taking the positive sign,

$$
I+V^{1 / 2} R_{0}(i \sigma)|V|^{1 / 2}=I+V^{1 / 2} R_{0}(i \sigma) V^{1 / 2}
$$

is a positive operator for $\sigma>0$. When we choose the negative sign, we will additionally assume that $\sigma$ is chosen large enough that $I-V^{1 / 2} R_{0}(i \sigma) V^{1 / 2}$ is a positive invertible operator; this is possible, for example, by insisting $\sigma>2\left(\|V\|_{\infty}+1\right)$ since $\left\|R_{0}(i \sigma)\right\| \leq 1 / \sigma^{2}$. With these assumptions on $\sigma$, using the properties of the determinant and the fact that $V \geq 0$ we may rewrite the function $F_{m, \pm V}(i \sigma)$ from (3.2) as

$$
\begin{aligned}
F_{m}(i \sigma)= & F_{m, \pm V}(i \sigma) \\
= & \operatorname{det}\left(I \pm i m\left(I \pm V^{1 / 2} R_{0}(i \sigma) V^{1 / 2}\right)^{-1 / 2} V^{1 / 2} T(i \sigma) V^{1 / 2}\right. \\
& \left.\left(I \pm V^{1 / 2} R_{0}(i \sigma) V^{1 / 2}\right)^{-1 / 2}\right) .
\end{aligned}
$$

We shall obtain a lower bound on $F_{m}(i \sigma)$ as $\sigma \rightarrow \infty$.

The following proposition is central to the proof of Theorem 1.1 and is the main result of this section. Related results were obtained in odd dimensions in $[4, \mathrm{Sec}-$ tion 5].

Proposition 4.1. Let $V \in L_{\mathrm{comp}}^{\infty}\left(\mathbb{R}^{d}\right), V \geq 0$, and let $V$ be bounded below by $\epsilon \chi_{B}$ where $\epsilon>0$ and $\chi_{B}$ is the characteristic function of a nontrivial open ball. Let $m \in \mathbb{Z}, m \neq 0$. Then there is a constant $c_{0}>0$ so that $\left|F_{m, \pm V}(i \sigma)\right| \geq$ $c_{0} \exp \left(c_{0} \sigma^{d}\right)$ for all sufficiently large $\sigma>0$.

The proof is similar to the proofs of some results of $[16,27]$ in that it uses both a property of monotonicity in $V$ and the fact that for potentials which are positive multiples of the characteristic function of a ball much can be said by using a decomposition into spherical harmonics and special functions. However, the implementation of these underlying ideas is rather different here.

The proof of Proposition 4.1 uses the following lemma, a monotonicity result reminiscent of results of $[16,27]$. In fact, the proof of this lemma uses a result from [27]. 
Lemma 4.2. Let $V_{1}, V_{2} \in L^{\infty}\left(\mathbb{R}^{d}\right)$ and suppose the support of $V_{j}$ is contained in $\bar{B}(R)=\left\{x \in \mathbb{R}^{d}:|x| \leq R\right\}$ for $j=1$, 2. Suppose $V_{2}(x) \geq V_{1}(x) \geq 0$ for all $x \in \mathbb{R}^{d}$. We use the convention that $V_{1}^{1 / 2} / V_{2}^{1 / 2}$ is 0 outside the support of $V_{1}$. Then for $\sigma>0$

$$
\left\|\left(I+V_{1}^{1 / 2} R_{0}(i \sigma) V_{1}^{1 / 2}\right)^{-1 / 2} \frac{V_{1}^{1 / 2}}{V_{2}^{1 / 2}}\left(I+V_{2}^{1 / 2} R_{0}(i \sigma) V_{2}^{1 / 2}\right)^{1 / 2}\right\| \leq 1 .
$$

If $\sigma \geq 2\left(\left\|V_{2}\right\|_{\infty}+1\right)$, then

$$
\left\|\left(I-V_{1}^{1 / 2} R_{0}(i \sigma) V_{1}^{1 / 2}\right)^{-1 / 2} \frac{V_{1}^{1 / 2}}{V_{2}^{1 / 2}}\left(I-V_{2}^{1 / 2} R_{0}(i \sigma) V_{2}^{1 / 2}\right)^{1 / 2}\right\| \leq 1 .
$$

Proof. When $\sigma>0$ is sufficiently large that $I \pm V_{j}^{1 / 2} R_{0}(i \sigma) V_{j}^{1 / 2}$ is a positive operator,

$$
\begin{aligned}
(I & \left. \pm V_{j} R_{0}(i \sigma)\right) V_{j}^{1 / 2}\left(I \pm V_{j}^{1 / 2} R_{0}(i \sigma) V_{j}^{1 / 2}\right)^{-1} \\
& =V_{j}^{1 / 2}\left(I \pm V_{j}^{1 / 2} R_{0}(i \sigma) V_{j}^{1 / 2}\right)\left(I \pm V_{j}^{1 / 2} R_{0}(i \sigma) V_{j}^{1 / 2}\right)^{-1} \\
& =V_{j}^{1 / 2}
\end{aligned}
$$

Thus

$$
V_{j}^{1 / 2}\left(I \pm V_{j}^{1 / 2} R_{0}(i \sigma) V_{j}^{1 / 2}\right)^{-1} V_{j}^{1 / 2}=\left(I \pm V_{j} R_{0}(i \sigma)\right)^{-1} V_{j}, \quad j=1,2
$$

for $\sigma>0$ sufficiently large. Applying [27, Lemma 2.2], and using that $V_{2} \geq V_{1}$, we get

$$
\left(I+V_{2} R_{0}(i \sigma)\right)^{-1} V_{2} \geq\left(I+V_{1} R_{0}(i \sigma)\right)^{-1} V_{1} .
$$

When we take the “-” sign, again applying [27, Lemma 2.2],

$$
\left(I-V_{2} R_{0}(i \sigma)\right)^{-1} V_{2} \geq\left(I-V_{1} R_{0}(i \sigma)\right)^{-1} V_{1}
$$

when $\sigma>2\left(\left\|V_{2}\right\|_{\infty}+1\right)$. Here we note our convention differs somewhat from [27], in that we take $V_{j} \geq 0$. Summarizing,

$$
V_{2}^{1 / 2}\left(I \pm V_{2}^{1 / 2} R_{0}(i \sigma) V_{2}^{1 / 2}\right)^{-1} V_{2}^{1 / 2} \geq V_{1}^{1 / 2}\left(I \pm V_{1}^{1 / 2} R_{0}(i \sigma) V_{1}^{1 / 2}\right)^{-1} V_{1}^{1 / 2}
$$

when $\sigma>0$ (for the "+" sign) or $\sigma>2(\|V\|+1$ ) (for the "-" sign). For the remainder of the proof, we shall assume $\sigma>0$ satisfies these requirements and suppress the argument $i \sigma$.

Now let $\chi_{V_{2}}$ be the characteristic function of the support of $V_{2}$, recall

$$
V_{1}^{1 / 2} \chi_{V_{2}}=V_{1}^{1 / 2}
$$


and note that

$$
\chi_{V_{2}}\left(I \pm V_{2}^{1 / 2} R_{0} V_{2}^{1 / 2}\right)=\left(I \pm V_{2}^{1 / 2} R_{0} V_{2}^{1 / 2}\right) \chi_{V_{2}} .
$$

Then

$$
\chi_{V_{2}}\left(I \pm V_{2}^{1 / 2} R_{0} V_{2}^{1 / 2}\right)^{-1} \chi_{V_{2}} \geq \frac{V_{1}^{1 / 2}}{V_{2}^{1 / 2}}\left(I \pm V_{1}^{1 / 2} R_{0} V_{1}^{1 / 2}\right)^{-1} \frac{V_{1}^{1 / 2}}{V_{2}^{1 / 2}} .
$$

This implies

$\chi_{V_{2}} \geq\left(I \pm V_{2}^{1 / 2} R_{0} V_{2}^{1 / 2}\right)^{1 / 2} \frac{V_{1}^{1 / 2}}{V_{2}^{1 / 2}}\left(I \pm V_{1}^{1 / 2} R_{0} V_{1}^{1 / 2}\right)^{-1} \frac{V_{1}^{1 / 2}}{V_{2}^{1 / 2}}\left(I \pm V_{2}^{1 / 2} R_{0} V_{2}^{1 / 2}\right)^{1 / 2}$.

This proves the lemma, since the norm of the right hand side is the square of the norm of the operator in question.

Lemma 4.3. Let $\mathcal{H}$ be an infinite dimensional complex separable Hilbert space, $A, B \in \mathcal{L}(\mathcal{H})$, with $B=B^{*}$, and $\|A\| \leq 1$. Let $\left|\lambda_{1}\right| \geq\left|\lambda_{2}\right| \geq \ldots$ be the norms of the eigenvalues of $A^{*} B A$, and $\left|\mu_{1}\right| \geq\left|\mu_{2}\right| \geq \ldots$ be the norms of the eigenvalues of $B$. In both cases we repeat according to multiplicity. Then $\left|\mu_{j}\right| \geq\left|\lambda_{j}\right|$ for all $j$.

Proof. One way to prove this it that by noting that since $B$ and $A^{*} B A$ are selfadjoint, the norms of the the eigenvalues are the characteristic values. Then this lemma is an immediate application of the bound for the characteristic values of a product found, for example, in [25, Theorem 1.6].

The next lemma shows that $F_{m, \pm V}(i \sigma)$ depends monotonically on $V$ in some sense.

Lemma 4.4. Let $V_{1}, V_{2} \in L^{\infty}\left(\mathbb{R}^{d}\right)$ and suppose the support of $V_{j}$ is contained in $\bar{B}(R)$ for $j=1$, 2. Suppose $V_{2}(x) \geq V_{1}(x) \geq 0$ for all $x \in \mathbb{R}^{d}$. Then

$$
\left|F_{m, V_{1}}(i \sigma)\right| \leq\left|F_{m, V_{2}}(i \sigma)\right| \quad \text { for all } \sigma \in \mathbb{R}_{+} .
$$

Moreover, if $\sigma \geq 2\left(\left\|V_{2}\right\|_{\infty}+1\right)$, then

$$
\left|F_{m,-V_{1}}(i \sigma)\right| \leq\left|F_{m,-V_{2}}(i \sigma)\right| .
$$

Proof. For any compactly supported $V \geq 0$, set

$$
\begin{gathered}
B_{1, \pm, V}(i \sigma)=\left(I \pm V^{1 / 2} R_{0}(i \sigma) V^{1 / 2}\right)^{-1 / 2} V^{1 / 2} T(i \sigma) V^{1 / 2} \\
\left(I \pm V^{1 / 2} R_{0}(i \sigma) V^{1 / 2}\right)^{-1 / 2}
\end{gathered}
$$


and notice that if $\sigma>0$ (for the " + " sign) or $\sigma>2\left(\|V\|_{\infty}+1\right)$ (for the "-" sign), $B_{1, \pm, V}(i \sigma)$ is a self-adjoint trace class operator. Comparing (4.1), we see that

$$
F_{m, \pm V}(i \sigma)=\operatorname{det}\left(I \pm i m B_{1, \pm, V}(i \sigma)\right) .
$$

Hence for sufficiently large $\sigma$

$$
\begin{aligned}
\left|F_{m, \pm V}(i \sigma)\right| & =\left|\prod\left(I+i m \lambda_{j}\left(B_{1, \pm, V}(i \sigma)\right)\right)\right| \\
& =\prod\left|\left(I+i m \lambda_{j}\left(B_{1, \pm, V}(i \sigma)\right)\right)\right| \\
& =\prod \sqrt{1+m^{2} \lambda_{j}^{2}\left(B_{1, \pm, V}(i \sigma)\right)}
\end{aligned}
$$

where $\lambda_{j}\left(B_{1, \pm, V}\right)$ are the nonzero eigenvalues of $B_{1, \pm, V}$, repeated according to multiplicity and arranged in decreasing order of magnitude:

$$
\left|\lambda_{1}\left(B_{1, \pm, V}\right)\right| \geq\left|\lambda_{2}\left(B_{1, \pm, V}\right)\right| \geq \ldots
$$

Now we turn to $V_{1}$ and $V_{2}$, and $\sigma$ as in the statement of the lemma. Note that

$$
\begin{aligned}
& B_{1, \pm, V_{1}}(i \sigma) \\
&=\left(I \pm V_{1}^{1 / 2} R_{0}(i \sigma) V_{1}^{1 / 2}\right)^{-1 / 2} \frac{V_{1}^{1 / 2}}{V_{2}^{1 / 2}}\left(I \pm V_{2}^{1 / 2} R_{0}(i \sigma) V_{2}^{1 / 2}\right)^{1 / 2} B_{1, \pm, V_{2}}(i \sigma) \\
&\left(I \pm V_{2}^{1 / 2} R_{0}(i \sigma) V_{2}^{1 / 2}\right)^{1 / 2} \frac{V_{1}^{1 / 2}}{V_{2}^{1 / 2}}\left(I \pm V_{1}^{1 / 2} R_{0}(i \sigma) V_{1}^{1 / 2}\right)^{-1 / 2} .
\end{aligned}
$$

Again we use the convention that $V_{1}^{1 / 2} / V_{2}^{1 / 2}$ is 0 outside the support of $V_{1}$. The lemma now follows from (4.3) and Lemmas 4.2 and 4.3.

In order to obtain the lower bounds of Proposition 4.1, we shall need a special case of that proposition, in which the potential is of the form $V(x)=\epsilon \chi_{B}(x)$, and $\chi_{B}(x)$ is the characteristic function of a ball centered at the origin. To study such a special case, we will introduce spherical coordinates in $\mathbb{R}^{d}$ (polar coordinates in the case $d=2$ ).

In spherical coordinates,

$$
-\Delta=-\frac{\partial^{2}}{\partial r^{2}}-\frac{d-1}{r} \frac{\partial}{\partial r}+\frac{1}{r^{2}} \Delta_{\mathbb{S}^{d-1}} .
$$

The eigenvalues of of the Laplacian on $\mathbb{S}^{d-1}, \Delta_{\mathbb{S}^{d-1}}$, are $l(l+d-2), l \in \mathbb{N}_{0}$ with multiplicity

$$
\mu(l)=\frac{2 l+d-2}{d-2}\left(\begin{array}{c}
l+d-3 \\
d-3
\end{array}\right)=\frac{2 l^{d-2}}{(d-2) !}\left(1+O\left(l^{-1}\right)\right) .
$$


Denote by $\left\{Y_{l}^{\mu}\right\}, 1 \leq \mu \leq \mu(l), l=0,1,2, \ldots$ a complete orthonormal set of spherical harmonics on $\mathbb{S}^{d-1}$ so that $\Delta_{\mathbb{S}^{d-1}} Y_{l}^{\mu}=l(l+d-2) Y_{l}^{\mu}$.

We denote by $P_{l}$ projection onto the span of

$$
\left\{h(|x|) Y_{l}^{\mu}(x /|x|): 1 \leq \mu \leq \mu(l), h \in L^{2}\left(\mathbb{R} ; r^{d-1} d r\right)\right\} .
$$

Thus writing $x=r \theta$, with $r>0$ and $\theta \in \mathbb{S}^{d-1}$

$$
\left(P_{l} g\right)(r \theta)=\sum_{\mu=1}^{\mu(l)} \int_{\mathbb{S}^{d-1}} g(r \omega) Y_{l}^{\mu}(\theta) \bar{Y}_{l}^{\mu}(\omega) d S_{\omega} .
$$

Lemma 4.5. Let $V \geq 0, V \in L_{\text {comp }}^{\infty}\left(\mathbb{R}^{d}\right)$ be a radial function, so that $V(x)=$ $f(|x|)$ for some function $f \in L_{\text {comp }}^{\infty}([0, \infty))$. Then for $\sigma>0$ sufficiently large, with $B_{1}=B_{1, \pm, V}$ the operator defined in (4.2),

$$
\left\|\left(V^{1 / 2} T(i \sigma) V^{1 / 2}-B_{1, \pm, V}(i \sigma)\right) P_{l}\right\| \leq \frac{C}{\sigma^{2}}\left\|V^{1 / 2} T(i \sigma) V^{1 / 2} P_{l}\right\|
$$

for a constant $C$ depending on $V$ but not $\sigma$ or $l$.

Proof. To simplify the notation, we write

$$
A(i \sigma)=A_{ \pm, V}(i \sigma)=I \pm V^{1 / 2} R_{0}(i \sigma) V^{1 / 2},
$$

and note that for $\sigma>0$ sufficiently large,

$$
\left\|A^{-1}(i \sigma)-I\right\|=O\left(1 / \sigma^{2}\right),\left\|A^{-1 / 2}(i \sigma)-I\right\|=O\left(1 / \sigma^{2}\right) .
$$

Now with $B_{1}$ the operator defined in (4.2),

$$
B_{1}-V^{1 / 2} T V^{1 / 2}=\left(A^{-1 / 2}-I\right) V^{1 / 2} T V^{1 / 2} A^{-1 / 2}+V^{1 / 2} T V^{1 / 2}\left(A^{-1 / 2}-I\right) .
$$

Because $V$ is radial, multiplication by either $V$ or $V^{1 / 2}$ commutes with $P_{l}$. Since $R_{0}$ commutes with $P_{l}$, so do $A, A^{-1}$, and $A^{-1 / 2}$. Thus

$$
\begin{gathered}
\left\|\left(B_{1}-V^{1 / 2} T V^{1 / 2}\right) P_{l}\right\| \\
\leq\left\|\left(A^{-1 / 2}-I\right)\right\|\left\|V^{1 / 2} T V^{1 / 2} P_{l}\right\|\left\|A^{-1 / 2}\right\| \\
\quad+\left\|V^{1 / 2} T V^{1 / 2} P_{l}\right\|\left\|\left(A^{-1 / 2}-I\right)\right\| .
\end{gathered}
$$

Thus using (4.4) we are done. 
Using the notation of [20], let $J_{v}$ and $Y_{v}$ denote the Bessel functions of the first and second kinds, respectively, and recall that $H_{v}^{(1)}(z)=J_{v(z)}+i Y_{v}(z)$. For $l \in \mathbb{N}_{0}$, set $v_{l}=l+(d-2) / 2$ and notice that $v_{l}$ is an integer since $d$ is even. We can now expand $R_{0}(\lambda)$ using spherical harmonics. When $0<\arg \lambda<\pi$ and $g \in L^{2}\left(\mathbb{R}^{d}\right)$,

$$
\begin{aligned}
& \left(R_{0}(\lambda) g\right)(r \theta) \\
& \quad=\sum_{l=0}^{\infty} \sum_{\mu=1}^{\mu(l)} \int_{0}^{\infty} \int_{\mathbb{S}^{d-1}} G_{v_{l}}\left(r, r^{\prime} ; \lambda\right) Y_{l}^{\mu}(\theta) \bar{Y}_{l}^{\mu}(\omega) g\left(r^{\prime} \omega\right)\left(r^{\prime}\right)^{d-1} d S_{\omega} d r^{\prime}
\end{aligned}
$$

with

$$
G_{v_{l}}\left(r, r^{\prime} ; \lambda\right)= \begin{cases}\frac{\pi}{2 i}\left(r r^{\prime}\right)^{-(d-2) / 2} J_{v_{l}}(\lambda r) H_{v_{l}}^{(1)}\left(\lambda r^{\prime}\right) & \text { if } r<r^{\prime}, \\ \frac{\pi}{2 i}\left(r r^{\prime}\right)^{-(d-2) / 2} H_{v_{l}}^{(1)}(\lambda r) J_{v_{l}}\left(\lambda r^{\prime}\right) & \text { if } r \geq r^{\prime} .\end{cases}
$$

As noted earlier, for compactly supported, bounded $\chi, \chi R_{0}(\lambda) \chi$ has an analytic continuation to $\Lambda$, and $G_{v_{l}}\left(r, r^{\prime} ; \lambda\right)$ does as well.

Now we use $[20,9.1 .35,9.1 .36]$ to obtain

$$
J_{v}\left(e^{i \pi} z\right)=e^{i \pi v} J_{v}(z) .
$$

Specializing $[20,9.1 .36]$ to the case of $v$ an integer we have

$$
Y_{v_{l}}\left(e^{i \pi} z\right)=e^{-v_{l} \pi i}\left(Y_{v_{l}}(z)+2 i J_{v_{l}}(z)\right)
$$

giving

$$
H_{v_{l}}^{(1)}\left(e^{i \pi} z\right)=e^{i v_{l} \pi}\left(-J_{v_{l}}(z)+i Y_{v_{l}}(z)\right)
$$

Thus

$$
\begin{aligned}
\widetilde{G}_{\nu_{l}}\left(r, r^{\prime} ; \lambda\right) & \stackrel{\text { def }}{=} G_{\nu_{l}}\left(r, r^{\prime} ; e^{i \pi} \lambda\right)-G_{\nu_{l}}\left(r, r^{\prime} ; \lambda\right) \\
& =i \pi\left(r r^{\prime}\right)^{-(d-2) / 2} J_{v_{l}}(\lambda r) J_{v_{l}}\left(\lambda r^{\prime}\right) .
\end{aligned}
$$

Together, (4.6) and (4.8) give us an expression for the Schwartz kernel of $R_{0}\left(e^{i \pi} \lambda\right)-R_{0}(\lambda)$ in spherical coordinates: with $r, r^{\prime}>0, \theta \in \mathbb{S}^{d-1}$,

$$
\begin{aligned}
& \left(\left(R_{0}\left(e^{i \pi \lambda}\right)-R_{0}(\lambda)\right) g\right)(r \theta) \\
& \quad=\sum_{l=0}^{\infty} \sum_{\mu=1}^{\mu(l)} \int_{0}^{\infty} \int_{\mathbb{S}^{d-1}} \widetilde{G}_{v_{l}}\left(r, r^{\prime} ; \lambda\right) Y_{l}^{\mu}(\theta) \bar{Y}_{l}^{\mu}(\omega) g\left(r^{\prime} \omega\right)\left(r^{\prime}\right)^{d-1} d S_{\omega} d r^{\prime} .
\end{aligned}
$$

We continue to denote by $P_{l}$ the operator given in (4.3). 
Lemma 4.6. Let $B_{1}$ be the operator defined in (4.2). Let $V_{0}=\epsilon \chi_{a}$, where $\epsilon, a>0$ and $\chi_{a}$ is the characteristic function of the ball of radius $a$ and center 0 . Fix a constant $M>3$. Then there is a constant $c>0$ independent of $\sigma$ so that

$$
\left\|B_{1, \pm, V_{0}}(i \sigma) P_{l}\right\| \geq c \frac{e^{c v_{l}}}{v_{l}}
$$

for all $l \in \mathbb{N}$ which satisfy $a \sigma / 6>v_{l}>a \sigma / M$ for all sufficiently large $\sigma>0$.

Before beginning the proof, we note that the constant $c$ does depend on $\epsilon$ and on $a$.

Proof. From Lemma 4.5 it suffices to prove an analogous lower bound for the quantity $\left\|V_{0}^{1 / 2} T(i \sigma) V_{0}^{1 / 2} P_{l}\right\|$.

Recall $i T(i \sigma)=R_{0}\left(e^{i \pi} i \sigma\right)-R_{0}(i \sigma)$. Set

$$
\psi_{l}(r \theta)=\chi_{a}(r \theta) Y_{l}^{\mu}(\theta) r^{-(d-2) / 2} J_{v_{l}}(i \sigma r)
$$

for any $\mu \in\{1, \ldots, \mu(l)\}$, and note that

$$
\left\|V_{0}^{1 / 2} T V_{0}^{1 / 2} P_{l}\right\| \geq \frac{\left|\left\langle V_{0}^{1 / 2} T V_{0}^{1 / 2} \psi_{l}, \psi_{l}\right\rangle\right|}{\left\|\psi_{l}\right\|^{2}} .
$$

By (4.8) and (4.9),

$$
\begin{aligned}
\frac{\left|\left\langle V_{0}^{1 / 2} T V_{0}^{1 / 2} \psi_{l}, \psi_{l}\right\rangle\right|}{\left\|\psi_{l}\right\|^{2}} & =\frac{\pi\left(\int_{0}^{a} \epsilon^{1 / 2}\left|J_{v_{l}}(i \sigma r)\right|^{2} r^{-(d-2)} r^{d-1} d r\right)^{2}}{\int_{0}^{a}\left|J_{\nu_{l}}(i \sigma r)\right|^{2} r^{-(d-2)} r^{d-1} d r} \\
& =\pi \epsilon \int_{0}^{a}\left|J_{\nu_{l}}(i \sigma r)\right|^{2} r d r \\
& \geq \pi \epsilon \int_{a / 2}^{a}\left|J_{v_{l}}(i \sigma r)\right|^{2} r d r .
\end{aligned}
$$

As in [20,9.6.3], setting

$$
I_{\nu}(z) \stackrel{\text { def }}{=} e^{-v \pi i / 2} J_{\nu}\left(z e^{i \pi / 2}\right), \quad-\pi<\arg z \leq \pi / 2,
$$

from $[20,9.7 .7]$ there is a constant $c>0$ so that for $v$ sufficiently large

$$
\left|I_{v}(v s)\right| \geq c \frac{e^{c v}}{\sqrt{v}}, \quad 3 \leq s \leq M .
$$


Here and below we denote by $c$ a positive constant, independent of $\nu, l$, and $\sigma$, which may change from line to line. Now we use that $\left|J_{v_{l}}(i \sigma z)\right|=\left|I_{v_{l}}(\sigma z)\right|$ and apply these to (4.10). We find that if $3 \leq \sigma r / \nu_{l} \leq M$ for all $r$ with $a / 2 \leq r \leq a$, then

$$
\left|\left\langle V_{0}^{1 / 2} T V_{0}^{1 / 2} \psi_{l}, \psi_{l}\right\rangle\right| \geq c \int_{a / 2}^{a} \frac{e^{2 v_{l} c}}{v_{l}} d r \geq c \frac{e^{2 v_{l} c}}{v_{l}}
$$

for all sufficiently large $\sigma$. Thus, this holds for $l$ satisfying $a \sigma / 6>v_{l}>a \sigma / M$ if $\sigma$ is sufficiently large, providing a lower bound on $\left\|V_{0}^{1 / 2} T V_{0}^{1 / 2} P_{l}\right\|$, and thus on $\left\|B_{1, \pm V_{0}}(i \sigma) P_{l}\right\|$.

Lemma 4.7. Let $V_{0}=\epsilon \chi_{a}$, where $\epsilon, a>0$ and $\chi_{a}$ is the characteristic function of the ball of radius $a$ and center 0 . Then for $m_{0} \neq 0, m_{0} \in \mathbb{Z}$, there is $a c>0$ so that for $\sigma>0$ sufficiently large

$$
F_{m_{0}, \pm V_{0}}(i \sigma) \geq c \exp \left(c \sigma^{d}\right)
$$

The constant $c$ depends on $a, \epsilon$, and $m_{0}$.

Proof. Recall that

$$
\left|F_{m_{0}, \pm V_{0}}(i \sigma)\right|=\left|\operatorname{det}\left(I \pm i m_{0} B_{1, \pm, V_{0}}(i \sigma)\right)\right|
$$

and that for sufficiently large $\sigma>0 B_{1}(i \sigma)$ is a self-adjoint operator. Thus for sufficiently large $\sigma$

$$
\left|F_{m_{0}, \pm V_{0}}(i \sigma)\right|=\prod_{j=1}^{\infty} \sqrt{1+m_{0}^{2} \lambda_{j}^{2}}
$$

where $\lambda_{j}$ are the nonzero eigenvalues of $B_{1, \pm, V_{0}}(i \sigma)$. The $\lambda_{j}$ of course depend on $\sigma$, but we omit this in our notation.

A decomposition of $B_{1, \pm, V_{0}}$ using spherical harmonics shows that $B_{1, \pm, V_{0}}$ has eigenvalue $\left\|B_{1, \pm, V_{0}} P_{l}\right\|$ with multiplicity (at least) $\mu(l)$. Thus using (4.11) and the fact that $\lambda_{j}^{2}>0$, we get

$$
\left|F_{m_{0}}(i \sigma)\right|^{2} \geq \prod_{l=1}^{\infty}\left(1+m_{0}^{2}\left\|B_{1, \pm, V_{0}} P_{l}\right\|^{2}\right)^{\mu(l)}
$$


for sufficiently large $\sigma$. From Lemma 4.6, we see

$$
\begin{aligned}
&\left|F_{m_{0}}(i \sigma)\right|^{2} \geq \prod_{a \sigma / 6>v_{l}>a \sigma / M}\left(1+c m_{0}^{2} \frac{e^{c v_{l}}}{v_{l}^{2}}\right)^{\mu(l)} \\
&=\exp \left(\sum_{a \sigma / 6>v_{l}>a \sigma / M} \mu(l) \log \left(1+c m_{0}^{2} \frac{e^{c v_{l}}}{v_{l}^{2}}\right)\right) \\
& \geq \exp \left(\sum_{a \sigma / 6-(d-2) / 2>l>a \sigma / M-(d-2) / 2} \mu(l)(c l-c(d-2) / 2\right. \\
&\left.\left.+\log \left(c m_{0}^{2} / v_{l}^{2}\right)\right)\right)
\end{aligned}
$$

Now for $l$ sufficiently large, $\mu(l) \geq l^{d-2} /(d-2)$ ! so we get

$$
\left|F_{m}(i \sigma)\right|^{2} \geq \exp \left(c \sigma^{d}-C\right)
$$

for some constants $C$ and $c>0$ for all sufficiently large $\sigma$.

Proof of Proposition 4.1. We are now ready to give the proof of Proposition 4.1. Since if $W$ is a translate of $V, F_{m, \pm, V}=F_{m, \pm, W}$, we may assume $V$ can be bounded below by $V_{0}=\epsilon \chi_{B_{a}}$, where $\chi_{B_{a}}$ is the characteristic function of the ball of radius $a>0$ and center at the origin. Then using Lemmas 4.4 and 4.7 proves the proposition immediately.

\section{Proof of Theorem 1.1}

Let $V \in L_{\text {comp }}^{\infty}\left(\mathbb{R}^{d}\right), V \geq 0$. We continue to assume $d$ is even and to use the function

$$
F_{m}(\lambda)=F_{m, \pm V}(\lambda)=\operatorname{det}\left(I \pm i m\left(1 \pm V^{1 / 2} R_{0}(\lambda) V^{1 / 2}\right)^{-1} V^{1 / 2} T(\lambda) V^{1 / 2}\right)
$$

defined first by (3.2). Note that since $\left(I \pm V^{1 / 2} R_{0}(\lambda) V^{1 / 2}\right)^{-1}$ is a meromorphic function on $\Lambda, F_{m, \pm V}(\lambda)$ is meromorphic on $\Lambda$. We shall be most interested in the behavior of $F_{m, \pm V}(\lambda)$ in $\bar{\Lambda}_{0}$, since the zeros of $F_{m, \pm V}$ in $\Lambda_{0}$ correspond to the poles of $R_{ \pm V}$ in $\Lambda_{m}$. In the proof of Theorem 1.1 we shall apply Proposition 2.4 to a function obtained by multiplying $F_{m, \pm V}$ by a rational function. Thus we begin this section by checking properties of $F_{m, \pm V}$.

Lemma 5.1. The function $F_{m, \pm V}(\lambda)$ has only finitely many poles in

$$
\{\lambda \in \Lambda: 0 \leq \arg \Lambda \leq \pi\}
$$

and only finitely many zeros with argument 0 or $\pi$. 
Proof. We recall first the well-known estimate

$$
\left\|V^{1 / 2} R_{0}(\lambda) V^{1 / 2}\right\| \leq C /|\lambda| \quad \text { for } \lambda \in \Lambda, 0 \leq \arg \lambda \leq \pi
$$

(e.g. [1, 28, 29]). Thus for $|\lambda| \geq 2 / C, I \pm V^{1 / 2} R_{0}(\lambda) V^{1 / 2}$ is invertible, with norm of the inverse bounded by 2 . Since the function $F_{m, \pm V}$ cannot have a pole at $\lambda_{0}$ unless $\left(I \pm V^{1 / 2} R_{0}(\lambda) V^{1 / 2}\right)^{-1}$ has a pole at $\lambda_{0}$, we see $F_{m, \pm V}(\lambda)$ has no poles in the region $\left\{\lambda \in \bar{\Lambda}_{0},|\lambda| \geq r_{0}\right\}$ for some constant $r_{0}$ depending on $V$.

Moreover, from (5.1) $\left\|V^{1 / 2} T(\lambda) V^{1 / 2}\right\| \leq C /|\lambda|$ for $\lambda \in \partial \bar{\Lambda}_{0}$. Thus, there is an $r_{0} \geq 0$ so that $F_{m, \pm V}(\lambda)$ has no zeros in $\left\{\lambda \in \partial \bar{\Lambda}_{0},|\lambda| \geq r_{0}\right\}$.

The bounds of Vodev [28, 29] ensure that there are only finitely many poles of $R_{ \pm V}(\lambda)$ in $\left\{\lambda \in \overline{\Lambda_{m}}:|\lambda| \leq r\right\}$ for any $r$. Since $F_{m, \pm V}$ has a pole at $\lambda \in \overline{\Lambda_{0}}$ only if $R_{ \pm V}$ has a pole there, and has a zero at $\lambda \in \partial \overline{\Lambda_{0}}$ only if $R_{ \pm V}$ has a pole at $e^{i m \pi} \lambda$, this finishes the proof of the claim.

Lemma 5.2. Let $t \in \Lambda$ have $\arg t=0$ or $\arg t=\pi$. Then there are constants $C$, $r_{0}>0$ depending on $V$ and $m$ so that

$$
\left|\frac{\frac{d}{d t} F_{m, \pm V}(t)}{F_{m, \pm V}(t)}\right| \leq C|t|^{d-2} \quad \text { for }|t| \geq r_{0} .
$$

Proof. Note that

$$
\frac{\frac{d}{d t} F_{m, \pm V}(t)}{F_{m, \pm V}(t)}=\operatorname{tr}\left( \pm i m(I \pm i m W(t))^{-1} \frac{d}{d t} W(t)\right)
$$

where

$$
W(t)=W_{ \pm V}(t)=\left(I \pm V^{1 / 2} R_{0}(t) V^{1 / 2}\right)^{-1} V^{1 / 2} T(t) V^{1 / 2} .
$$

Using (5.1) we see that that there is an $r_{0}>0$ so that

$$
\left\|\left(I \pm V^{1 / 2} R_{0}(t) V^{1 / 2}\right)^{-1}\right\| \leq 2 \text { for }|t|>r_{0} .
$$

For the values of $t$ in question (on the boundary of the physical region), for any $\chi \in C_{c}^{\infty}\left(\mathbb{R}^{d}\right)$ and any $j \in \mathbb{N}_{0}$ there are constants $C_{j}$ depending on $\chi$ so that

$$
\left\|\frac{d^{j}}{d t^{j}} \chi R_{0}(t) \chi\right\| \leq C_{j}|t|^{-1}, \quad|t| \geq 1,
$$

see e.g. [14, Section 8] or [15, Section 16]. This implies that for $|t|$ sufficiently large with $\arg t=0, \pi,\left\|\frac{d^{j}}{d t^{j}} W(t)\right\| \leq C_{j}, j=0,1$, for some new constant $C_{j}$ depending on $V$. 
Now we use an argument as in [12, Lemma 3.3] to bound both $\|W(t)\|_{1}$ and $\left\|\frac{d}{d t} W(t)\right\|_{1}$, where $\|\cdot\|_{1}$ is the trace class norm. We write, for $\chi \in L_{\text {comp }}^{\infty}\left(\mathbb{R}^{d}\right)$

$$
\chi T(\lambda) \chi=\alpha_{d} \lambda^{d-2} \mathbb{E}_{\chi}^{t}\left(e^{i \pi} \lambda\right) \mathbb{E}_{\chi}(\lambda)
$$

where

$$
\mathbb{E}_{\chi}(\lambda)(\theta, x)=\chi(x) e^{i \lambda x \cdot \theta}, \quad x \in \mathbb{R}^{d}, \theta \in \mathbb{S}^{d-1} .
$$

Then, just as in [12], we note that with $\|\cdot\|_{2}$ denoting the Hilbert-Schmidt norm,

$$
\left\|\mathbb{E}_{\chi}(t)\right\|_{2}^{2}=\int_{\mathbb{S}^{d-1}} \int_{\mathbb{R}^{d}}\left|e^{i t \omega \cdot x} \chi(x)\right|^{2} d x d \omega \leq C_{\chi}, \quad \text { for }(\arg t) / \pi \in \mathbb{Z}
$$

and

$\left\|\frac{d}{d t} \mathbb{E}_{\chi}(t)\right\|_{2}^{2}=\int_{\mathbb{S}^{d-1}} \int_{\mathbb{R}^{d}}\left|i(\omega \cdot x) e^{i t \omega \cdot x} \chi(x)\right|^{2} d x d \omega \leq C_{\chi}, \quad$ for $(\arg t) / \pi \in \mathbb{Z}$.

The same estimate holds for $\left\|\mathbb{E}_{\chi}^{t}\left(e^{i \pi} t\right)\right\|_{2}^{2}$ and $\left\|\frac{d}{d t} \mathbb{E}_{\chi}^{t}\left(e^{i \pi} t\right)\right\|_{2}^{2}$. Putting this all together and using that $\|A B\|_{1} \leq\|A\|_{2}\|B\|_{2}$, we see that

$$
\left\|\frac{d^{j}}{d t^{j}} W(t)\right\|_{1} \leq C\left(1+|t|^{d-2}\right), \quad \text { for } j=0,1 .
$$

Thus

$$
\begin{aligned}
\left|\frac{\frac{d}{d t} F_{m, \pm V}(t)}{F_{m, \pm V}(t)}\right| & =\left|\operatorname{tr}\left( \pm i m(I \pm i m W(t))^{-1} \frac{d}{d t} W(t)\right)\right| \\
& \leq\left\|m(I \pm i m W(t))^{-1} \frac{d}{d t} W(t)\right\|_{1} \\
& \leq C|t|^{d-2}
\end{aligned}
$$

when $|t|$ is sufficiently large.

The next lemma gives a bound on $F_{m, \pm V}(z), z \in \Lambda_{0}$, which is of a type which has been repeatedly used in proofs of upper bounds on the number of resonances. Closely related results can be found in $[18,32,12]$, among others. We include the proof for the convenience of the reader, although it is essentially a minor modification of arguments used in, for example, $[32,12]$ to, in the odd-dimensional case, bound something like the determinant of the scattering matrix in the physical half-plane. 
Lemma 5.3. There are constants $C, r_{0}>0$ depending on $V$ and $m$ so that

$$
\left|F_{m, \pm, V}(\lambda)\right| \leq C \exp \left(C|\lambda|^{d}\right), \quad \text { for all } \lambda \in \overline{\Lambda_{0}},|\lambda|>r_{0} .
$$

Proof. Using (5.5) and that $\operatorname{det}(I+A B)=\operatorname{det}(I+B A)$ when both $A B$ and $B A$ are trace class,

$$
F_{m, \pm, V}(\lambda)=\operatorname{det}(I+K(\lambda))
$$

where

$$
K(\lambda): L^{2}\left(\mathbb{S}^{d}\right) \longrightarrow L^{2}\left(\mathbb{S}^{d}\right)
$$

is given by

$$
K(\lambda)= \pm i m \alpha_{d} \lambda^{d-2} \mathbb{E}_{V^{1 / 2}}(\lambda)\left(I \pm V^{1 / 2} R_{0}(\lambda) V^{1 / 2}\right)^{-1} \mathbb{E}_{V^{1 / 2}}^{t}\left(e^{i \pi} \lambda\right) .
$$

Choose $r_{0} \geq 0$ so that

$$
\left\|\left(I \pm V^{1 / 2} R_{0}(\lambda) V^{1 / 2}\right)^{-1}\right\| \leq 2 \text { for } \lambda \in \Lambda_{0},|\lambda| \geq r_{0} .
$$

By slight abuse of notation, we denote the Schwartz kernel of $K$ by $K$ as well. Then there is some constant $C$ so that for each $j \in \mathbb{N}$,

$$
\left|\Delta_{\mathbb{S}^{d-1}, \theta}^{j} K(\lambda)(\theta, \omega)\right| \leq C^{2 j+1}\left(|\lambda|^{2 j}+(2 j) !\right) e^{C|\lambda|} \text { for } \lambda \in \overline{\Lambda_{0}},|\lambda| \geq r_{0}
$$

since

$$
\left.\left|\Delta_{\mathbb{S}^{d-1}}^{k} e^{i \lambda x \cdot \theta} V^{1 / 2}(x)\right| \leq C^{k}\left(|\lambda|^{2 k}+(2 k) !\right)\right) e^{C|\lambda|}
$$

and

$$
\left|\left(I \pm V^{1 / 2} R_{0}(\lambda) V^{1 / 2}\right)^{-1} \mathbb{E}_{V^{1 / 2}}\left(e^{i \pi} \lambda\right)^{t}\right| \leq C \exp (C|\lambda|),
$$

when $|\lambda| \geq r_{0}$. Thus by [32, Proposition 2],

$$
|\operatorname{det}(I+K(\lambda))| \leq C^{\prime} e^{C^{\prime}|\lambda|^{d}}, \quad \lambda \in \overline{\Lambda_{0}},|\lambda|>r_{0} .
$$

We are now ready to give the proof of Theorem 1.1.

Proof. The proof is by contradiction. So suppose for some fixed potential $V$ satisfying the hypotheses of the theorem and for some value of $m \in \mathbb{Z} \backslash\{0\}$ and for choice of sign (positive or negative)

$$
\lim \sup _{r \rightarrow \infty} \frac{\log n_{m, \pm V}(r)}{\log r}<d .
$$

We work with this fixed value of $m$ and fixed choice of sign for the remainder of this proof. For this choice of $m$ and sign consider the function

$$
F_{m, \pm V}(\lambda)=\operatorname{det}\left(I \pm i m\left(1 \pm V^{1 / 2} R_{0}(\lambda) V^{1 / 2}\right)^{-1} V^{1 / 2} T(\lambda) V^{1 / 2}\right) .
$$


We denote by $\tilde{n}(r)$ the number of zeros, counted with multiplicity, of $F_{m, \pm V}$ in $\Lambda_{0}$ of norm at most $r$. The assumption (5.6) means that there is a constant $d^{\prime}<d$ so that $n_{m, \pm V}(r)=O\left(r^{d^{\prime}}\right)$ for $r \rightarrow \infty$. Since with at most finitely many exceptions the zeros of $F_{m, \pm V}$ in $\Lambda_{0}$ correspond, with multiplicity, to the poles of $R_{ \pm V}$ in $\Lambda_{m}$ (see Section 3), $\tilde{n}(r)=n_{m, \pm V}(r)+O(1) \leq C\left(1+r^{d^{\prime}}\right)$ for some constant $C$.

We identify $\Lambda_{0}$ with the upper half plane and use the variable $z$ there. Thus we may think of $F_{m, \pm V}$ as function meromorphic in a neighborhood of

$$
\Omega=\{z \in \mathbb{C}:|z| \geq 1,0 \leq \arg z \leq \pi\} .
$$

Let $a_{1}, \ldots, a_{m_{p}}$ be the poles of $F_{m, \pm V}$ in $\Omega$, and let $b_{1}, \ldots, b_{m_{\zeta}}$ be the zeros of $F_{m, \pm V}$ in $\partial \Omega$, in both cases repeated according to multiplicity. Recall we know there are only finitely many by Lemma 5.1. Now set

$$
h(z) \stackrel{\prod_{\text {def }}^{=}}{\prod_{j=1}^{m_{p}}\left(z-a_{j}\right)} F_{m, \pm V}(z) .
$$

If there are no poles or no real zeros, the corresponding product is omitted. By applying Lemmas 5.2 and 5.3, we see that $h$ satisfies the hypotheses of Proposition 2.4 with $\rho=\max \left(d^{\prime}, d-1+\epsilon\right)$ for any $\epsilon>0$. Thus for some constant $C$, $\left|F_{m, \pm V}(z)\right| \leq C \exp \left(C|z|^{\rho}\right)$ for $z \in \Omega$ and $\rho<d$. But this contradicts Proposition 4.1.

\section{References}

[1] S. Agmon, Spectral properties of Schrödinger operators and scattering theory. Ann. Scuola Norm. Sup. Pisa Cl. Sci. (4) 2 (1975), no. 2, 151-218. MR 0397194 Zbl 0315.47007

[2] A. Autin, Isoresonant complex-valued potentials and symmetries. Canad. J. Math. 63 (2011), no. 4, 721-754. MR 2848996 Zbl 1220.31012

[3] L.-H. Chen, A sub-logarithmic lower bound for resonance counting function in two-dimensional potential scattering. Rep. Math. Phys. 65 (2010), no. 2, 157-164. MR 2674840 Zbl 1218.81101

[4] T. Christiansen, Several complex variables and the distribution of resonances in potential scattering. Comm. Math. Phys. 259 (2005), no. 3, 711-728. MR 2174422 Zbl 1088.81093 
[5] T. Christiansen, Schrödinger operators with complex-valued potentials and no resonances. Duke Math. J. 133 (2006), no. 2, 313-323. MR 2225694 Zbl 1107.35094

[6] T. Christiansen, Isophasal, isopolar, and isospectral Schrödinger operators and elementary complex analysis. Amer. J. Math. 130 (2008), no. 1, 49-58. MR 2382141 Zbl 1140.35005

[7] T. J. Christiansen and P. D. Hislop, Maximal order of growth for the resonance counting functions for generic potentials in even dimensions. Indiana Univ. Math. J. 59 (2010), no. 2, 621-660. MR 2648080 Zbl 1202.81199

[8] T. J. Christiansen and P. D. Hislop, Some remarks on resonances in even-dimensional Euclidean scattering. Preprint 2013. arXiv:1307.5822 [math-ph]

[9] J. Conway, Functions of one complex variable. Second edition. Graduate Texts in Mathematics, 11. Springer Verlag, Berlin etc., 1978. MR 0503901

[10] T.-C. Dinh and D.-V. Vu, Asymptotic number of scattering resonances for generic Schrödinger operators. Comm. Math. Phys. 326 (2014), no. 1, 185-208. MR 3162489 Zbl 1294.47016

[11] R. Froese, Asymptotic distribution of resonances in one dimension. J. Differential Equations 137 (1997), no. 2, 251-272. MR 1456597 Zbl 0955.35057

[12] R. Froese, Upper bounds for the resonance counting function of Schrödinger operators in odd dimensions. Canad. J. Math. 50 (1998), no. 3, 538-546. MR 1629819 Zbl 0918.47005

[13] A. Intissar, A polynomial bound on the number of the scattering poles for a potential in even-dimensional spaces $\mathbb{R}^{n}$. Comm. Partial Differential Equations 11 (1986), no. 4, 367-396. MR 0829322 Zbl 0607.35069

[14] A. Jensen and T. Kato, Spectral properties of Schrödinger operators and timedecay of the wave functions. Duke Math. J. 46 (1979), no. 3, 583-611. MR 0544248 Zbl 0448.35080

[15] A. Komech and E. Kopylova, Dispersion decay and scattering theory. John Wiley \& Sons, Hoboken, N.J., 2012. MR 3015024 Zbl 06042927

[16] P.D. Lax and R. S. Phillips, Decaying modes for the wave equation in the exterior of an obstacle. Comm. Pure Appl. Math. 22 (1969), 737-787. MR 0254432 Zbl 0181.38201

[17] B. Ja. Levin, Distribution of zeros of entire functions. American Mathematical Society, Providence, R.I., 1964. MR 0156975 Zbl 0152.06703

[18] R. B. Melrose, Polynomial bound on the number of scattering poles. J. Funct. Anal. 53 (1983), no. 3, 287-303. MR 0724031 Zbl 0535.35067

[19] R. B. Melrose, Geometric scattering theory. Stanford Lectures. Cambridge University Press, Cambridge, 1995. MR 1350074 Zbl 0849.58071

[20] F. W. J. Olver, Bessel functions of integer order. In M. Abramowitz and I. Stegun (eds.), Handbook of mathematical functions with formulas, graphs, and mathematical tables. National Bureau of Standards Applied Mathematics Series, 55. Government Printing Office, Washington, DC, 1964, 355-434. MR 0167642 (collection) Zbl 0171.38503 (collection) 
[21] T. Regge, Analytic properties of the scattering matrix. Nuovo Cimento 8 (5), (1958), 671-679. MR 0095702 Zbl 0080.41903

[22] A. Sá Barreto, Lower bounds for the number of resonances in even dimensional potential scattering. J. Funct. Anal. 169 (1999), 314-323. MR 1726757 Zbl 0939.35133

[23] A. Sá Barreto and S.-H. Tang, Existence of resonances in even dimensional potential scattering. Comm. Partial Differential Equations 25 (2000), no. 5-6, 1143-1151. MR 1759805 Zbl 0947.35101

[24] B. Simon, Resonances in one dimension and Fredholm determinants. J. Funct. Anal. 178 (2000), no. 2, 396-420. MR 1802901 Zbl 0977.34075

[25] B. Simon, Trace ideals and their applications. $2^{\text {nd }}$ ed. Mathematical Surveys and Monographs, 120. American Mathematical Society, Providence, R.I., 2005. MR 2154153 Zbl 1074.47001

[26] P. Stefanov, Sharp upper bounds on the number of the scattering poles. J. Funct. Anal. 231 (2006), no. 1, 111-142. MR 2190165 Zbl 1099.35074

[27] A. Vasy, Scattering poles for negative potentials. Comm. Partial Differential Equations 22 (1997), no. 1-2, 185-194. MR 1434143 Zbl 0884.35109

[28] G. Vodev, Sharp bounds on the number of scattering poles in even-dimensional spaces. Duke Math. J. 74 (1994), no. 1, 1-17. MR 1271461 Zbl 0813.35075

[29] G. Vodev, Sharp bounds on the number of scattering poles in the two-dimensional case. Math. Nachr. 170 (1994), 287-297. MR 1302380 Zbl 0829.35091

[30] M. Zworski, Distribution of poles for scattering on the real line. J. Funct. Anal. 73 (1987), no. 2, 277-296. MR 0899652 Zbl 0662.34033

[31] M. Zworski, Sharp polynomial bounds on the number of scattering poles of radial potentials. J. Funct. Anal. 82 (1989), 370-403. MR 0987299 Zbl 0681.47002

[32] M. Zworski, Sharp polynomial bounds on the number of scattering poles. Duke Math. J. 59 (1989), no. 2, 311-323. MR 1016891 Zbl 0681.47002

Received February 20, 2014; revised July 9, 2014

T. J. Christiansen, Department of Mathematics, 202 Mathematical Sciences Building, University of Missouri, Columbia, Missouri 65211, USA

e-mail: christiansent@missouri.edu 